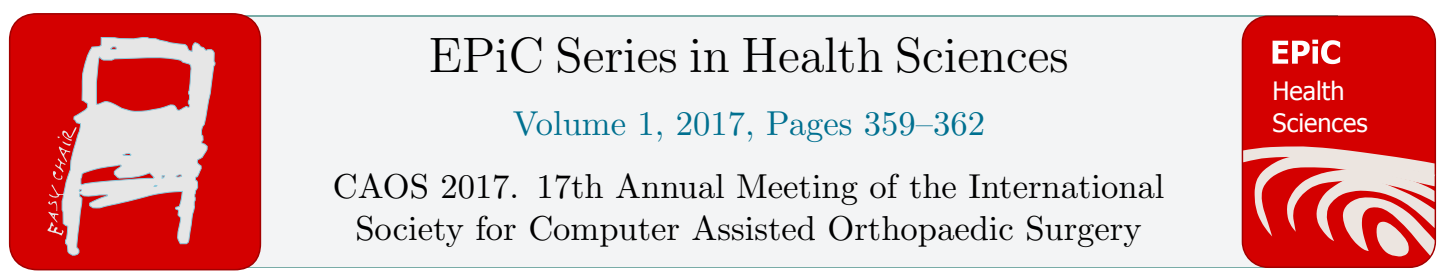

\title{
Pelvic rotation and inclination affect reliability of sagittal pelvic alignment and anterior pelvic plane angle measured by EOS system
}

\author{
Masashi Okamoto $^{1 *}$ and Kunihiko Tokunaga ${ }^{1 \dagger}$ \\ ${ }^{1}$ Niigata Hip Joint Center, Kameda Daiichi Hospital, Niigata, Japan. \\ rock. annonymous@gmail. com
}

\begin{abstract}
Background: The Difficulties to obtain acceptable pelvic reference points on biplanar X-ray images for EOS measurements change as the patient's pelvis rotated or inclined. The aim of this study was to clarify the effects of pelvic Z-axis rotation (rotation) and X-axis rotation (inclination) on the measurement values of SS, PT, and APP angle by using of EOS. Methods: We obtained anteroposterior and lateral X-ray images of a trunk phantom which was rotated and inclined in standing position by using of the EOS. Sacral slope (SS) and pelvic tilt (PT) as parameters to judge patient's pelvic alignments by spinal surgeons, and anterior pelvic (APP) angle as a reference to decide patient's pelvic alignments by hip surgeons were measured with the EOS software. The values of SS and PT and APP angle were compared in the phantom rotation and inclination. Results: PT showed small changes when the phantom was rotated, however, SS decreased and APP angle increased when the phantom was rotated. The values of SS and PT dispersed at $30^{\circ}$ rotation among each inclination. APP angle dispersed at $-15^{\circ}$ and $30^{\circ}$ of rotation among each inclination. Conclusion: Measurement variations of SS and PT were small amount under 15 degrees of rotation and inclination. On the contrary, APP angles showed greater variations when the phantom was rotated and inclined. Proper positioning of subjects, by reducing rotational effects, is important to get constant reproducible measurements, even if we use EOS system.
\end{abstract}

\section{Introduction}

The EOS imaging system (EOS system) (EOS imaging, Paris, France) provides reconstructed three dimensional coordinate based on anteroposterior and lateral X-ray images when we analyze these bi-planar images. Acceptable intra- and inter-observer reliability of the modality was reported

${ }^{*}$ Created the first draft of this document, Corresponding Author

${ }^{\dagger}$ Mentor for writing of this document 
previously [1]. Spinal surgeons often use sacral slope (SS) and pelvic tilt (PT) as parameters to judge patients' pelvic alignments [2]. On the other hand, hip surgeons use anterior pelvic plane (APP) angle as a reference to decide patients' pelvic alignments [3]. The coordinate axis of EOS measurements was arranged by correcting the axis between the bilateral patient's acetabular centers to be parallel to the axis of the modality. Therefore, the EOS images were not affected by patients' positions during image acquisition [4]. However, we sometimes experienced difficulties to obtain acceptable pelvic reference points on bi-planar X-ray images in EOS measurements when patient's pelvis rotated and inclined. The aim of this study was to clarify the effects of pelvic Z-axis rotation (rotation) and X-axis rotation (inclination) on the measurement values of SS, PT, and APP angle by using of EOS system.

\section{Materials and Methods}

We obtained anteroposterior and lateral X-ray images of a trunk phantom (Kyotokagaku, Kyoto, Japan) which was rotated and inclined in standing position by using of EOS system. The rotation axis was the Z-axis of the three dimensional coordinate in the EOS gantry, and we defined left rotation of the phantom as positive rotation. The inclination axis was the $\mathrm{X}$-axis of the same coordinate as described above. We defined anterior inclination (anteversion) of the phantom as positive inclination, and posterior inclination (retroversion) as negative one. SS, PT, and APP angle (the angles between the vertical line and the APP line corresponded to the line between the pubic symphysis and the midpoint between the two anterior superior iliac crests) were measured with the EOS software. The single examiner (Okamoto $\mathrm{M}$ ) measured each pelvic alignment parameter 5 times and analyze the effects of the phantom rotation and inclination on the measurement values. Inclination angles (15 degrees) were added to the values of SS, PT, and APP angle when the phantom was in retroversion, and were subtracted from the values of SS, PT, and APP angle when the phantom was in anteversion to make plots in Figure 1.

\section{Results}

The mean values of the measured angular parameters were shown in Figure 1. The mean values of SS with rotation at $-30^{\circ} / 0^{\circ} / 30^{\circ}$ were $31.9^{0} \pm 1.0^{\circ} / 37.3^{\circ} \pm 0.5^{\circ} / 32.2^{\circ} \pm 0.8^{0}$, respectively. The mean value of PT with rotation at $-30^{\circ} / 0^{0} / 30^{0}$ were $17.3^{\circ} \pm 0.3^{0} / 16.7^{0} \pm 0.3^{0} / 19.4^{0} \pm 0.4^{0}$, respectively. The mean values of APP angle with rotation at $-30^{\circ} / 0^{\circ} / 30^{\circ}$ were $13.8^{0} \pm 1.7^{0} / 4.6^{0} \pm$

$0.7^{0} / 13.4^{0} \pm 1.9^{0}$, respectively. PT showed small changes when the phantom was rotated, however, SS decreased and APP angle increased when the phantom was rotated. The APP angles changed dramatically when the phantom was rotated. The values of SS and PT dispersed at $30^{\circ}$ rotation among each inclination. APP angle dispersed at $-15^{\circ}$ and $30^{\circ}$ of rotation among each inclination. 
Pelvic Rotation and Inclination Affect Reliability of Sagittal Pelvic ... M. Okamoto and K. Tokunaga

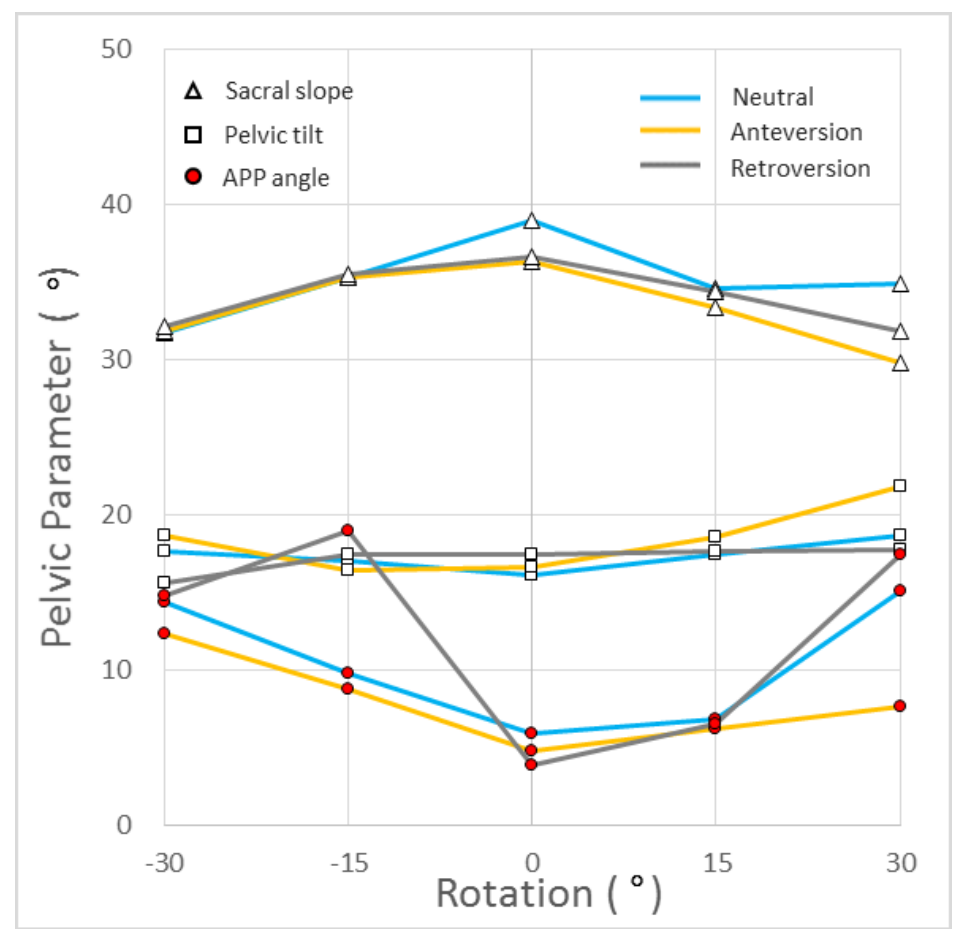

Figure 1: The mean values of SS, PT, and APP angle in each inclination and rotation

\section{Discussion}

Our results demonstrated influences of subject's rotation on the measurement values of SS, PT and APP angle by using of EOS system. Measurement variations of SS and PT were small amount under 15 degrees of rotation and inclination. On the contrary, APP angles showed greater variations when the phantom was rotated and inclined. PT showed lowest effect on rotation. The effect of Z-axis rotation reduced because the mid-point between the femoral head centers was used for the measurement of PT. SS also showed lower effects on rotation. The line of superior sacral plate on the sagittal plane was used to measure SS [5]. The effect of rotation was small because the length of the sacral plate was relatively short. The effect of rotation was significant on measurement values of APP angles. To measure APP angle, we had to identify three anatomical landmarks (bilateral superior iliac spines and symphysis pubis) on bi-planar X-ray images. To visualize anatomical landmarks on radiographs was not always easy [6]. Figure 2 showed difficulties to identify the anterior superior iliac spines and antero-superior point of the pubic symphysis when the subject was rotated. In addition, the distance of landmarks for APP angle measurement were larger than the other distances of landmarks for PT and SS measurements, therefore the effect of rotation was significant on APP angle.

Even though EOS provides non-distorted fine images due to its slit scanner and high sensitive detector, the operator can use the reference points only on the two dimensional images. In another words, we cannot set up new reference points on the three dimensional statistical pelvic model which created based on the bi-planer X-ray images in EOS system. In conclusion, proper positioning of subjects, by reducing rotational effects, is important to get constant reproducible measurements, even if we use EOS system. 


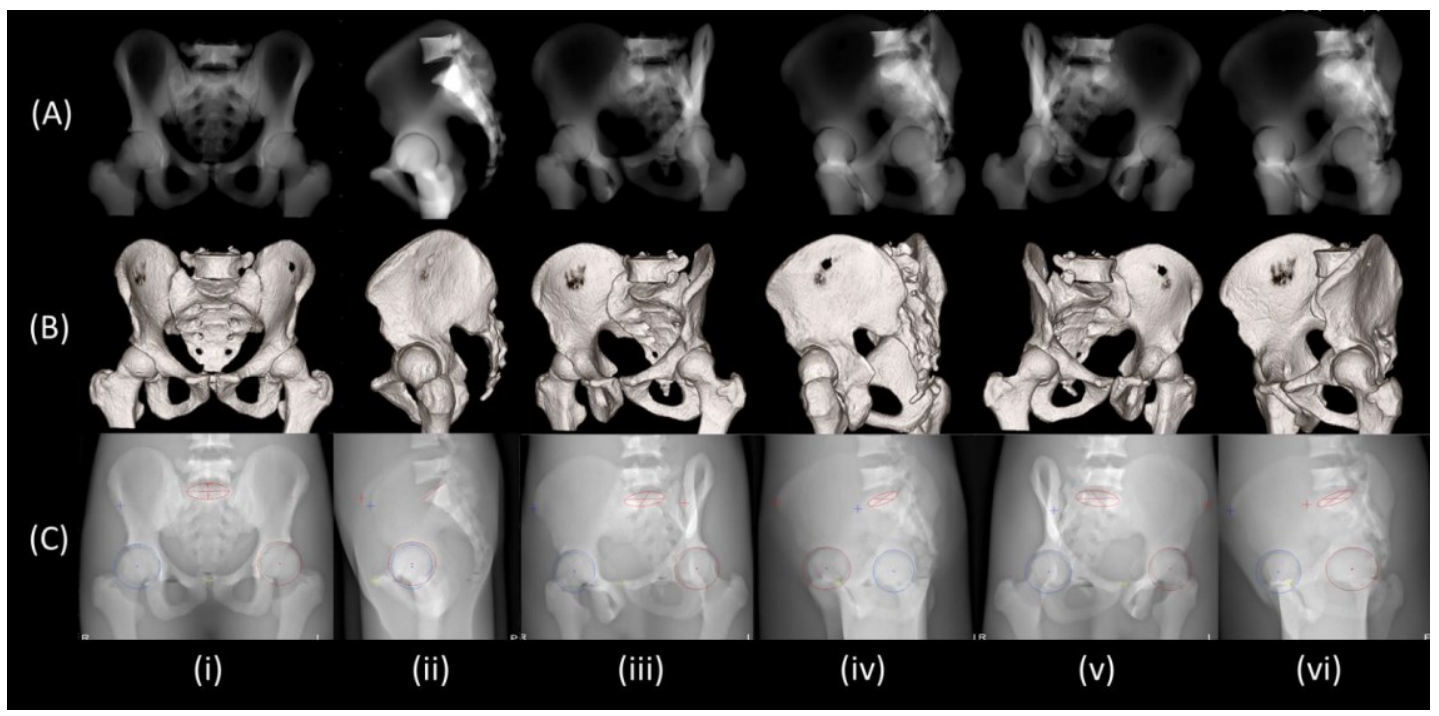

Figure 2: (A) Multiplanar reconstruction (MPR) images of the pelvis reconstructed with the CT software. (B) Three dimensional images of the pelvis reconstructed with the CT software. (C) Slot radiography images of the pelvis with modeling plot point for measures APP angle by EOS software. ( $i$, ii ) Coronal and sagittal images at 0 degree rotation. ( iii, iv ) Coronal and sagittal images at -30 degrees rotation. ( $v$, vi ) Coronal and sagittal images at 30 degrees rotation at 30 degrees rotation.

\section{References}

1. Glaser DA, Doan J, Newton PO, et al. Comparison of 3-dimensional spinal reconstruction accuracy, Spine, 37(16), pp: 1391-1397, 2012.

2. Lazennec JY, Brusson A, Rousseau MA, et al. Hip-Spine relation and sagittal balance clinical consequence, Eur Spine J, 5, pp:686-698, 2011.

3. Tamura S, Nishihara S, et al. Does pelvic sagittal inclination in the supine and standing positions change over 10 years of follow-up after total hip arthroplasty ?, J Arthroplasty. 35 pp: 1-6, 2016.

4. Carreau JH, Bastrom T, Petcharaporn M, et al. Computer-generated, three-dimensional spine model from biplanar radiographs: a validity study in idiopathic scoliosis curves greater than 50 degrees, Spine Deformity, 2, pp:81-88, 2014.

5. Legaye J, Duval-Beaupère G, Hecquet $\mathrm{J}$, Marty $\mathrm{C}$, et al. Pelvic incidence: a fundamental pelvic paramet

6. Vaz G, Roussouly P, Berthonnaud E, et al. Sagittal morphology and equilibrium of pelvis and spine, Eur Spine J, 11, pp:80-87, 2002. 